\title{
Synchronization of multiscale waveform focusing for rogue wave generation in dust acoustic wave turbulence
}

\author{
Po-Cheng Lin (1) and Lin I (1) \\ Department of Physics and Center for Complex Systems, National Central University, Jhongli, Taiwan 32001, Republic of China
}

(Received 16 December 2019; accepted 13 March 2020; published 28 April 2020)

\begin{abstract}
Rogue wave events (RWEs), uncertainly emerging localized extreme-amplitude events, ubiquitously occur in various nonlinear wave media. They have been mainly studied in the weakly disordered wave state with a single dominant wavelength scale. In this work, beyond the conventional stability approach, we experimentally investigate the rogue wave formation in the three-dimensional (3D) multiscale dust acoustic wave turbulence from the views of spatiotemporal waveform dynamics and the wave-particle interaction of multiscale modes. By decomposing turbulent waveforms into different-scale modes through multidimensional empirical mode decomposition, we demonstrate that RWE formation is associated with the spatiotemporal synchronization of multiscale wave crests and their envelope peaks. The synchronized 3D particle focusing by preceding distorted waveforms near low-amplitude holes of different modes is the key leading to RWE generation.
\end{abstract}

DOI: 10.1103/PhysRevResearch.2.023090

\section{INTRODUCTION}

Unraveling the generic dynamical behaviors of extreme events and their generation, which ubiquitously occurs in various complex systems such as temperature and rainfall in climate systems, seismic systems, financial systems, and nonlinear wave systems, is fundamentally challenging. In nonlinear wave systems, such as water surfaces [1,2], nonlinear optics [3], chemical waves [4], acoustic waves [5], and plasmas waves [6-9], increasing external drive can lead to the wave turbulence state exhibiting multiscale fluctuations and a continuous power spectrum. This feature makes wave turbulence a paragon to study the generic behaviors of the extreme events generated through the multiscale wave excitations and the associated waveform dynamics.

In nonlinear waves, uncertainly emerging localized largeamplitude events are named rogue waves. They have been observed in water surface waves [10-14], nonlinear optical waves $[3,15,16]$, second sounds of superfluid He II [5], ion acoustic waves [17], and dust acoustic wave systems [18]. Modulation instability, which causes waveform modulation and the formation of soliton-like envelopes, has been widely accepted as the basic mechanism for generating rogue wave events (RWEs) at envelope soliton peaks, in systems governed by nonlinear equations such as nonlinear LandauGinzburg and Schrödinger equations [10-13,15-17]. However, the above approach has been mainly limited to the one-dimensional (1D) weakly disordered wave with a single dominant frequency (wavelength) scale exhibiting distinct

Published by the American Physical Society under the terms of the Creative Commons Attribution 4.0 International license. Further distribution of this work must maintain attribution to the author(s) and the published article's title, journal citation, and DOI. peaks (fundamental and harmonics) in its power spectrum $[11-13,15,17]$. The generation mechanism for the multiscale wave turbulence state with a continuous power spectrum, especially from the wave-particle interaction view in the highdimensional system, still remains a fundamental unexplored issue.

Mechanical waves are constituted by particles. From the wave-particle interaction view, particle motion is affected by the wave field determined by the waveform, which consequently affects the waveform evolution [14,18-20]. For the higher dimensional weakly disordered wave with a single dominant wavelength scale, the focusing of particles by the distorted preceding surrounding waveform as the key for RWE generation, were demonstrated in the recent studies in two-dimensional (2D) water surface waves [14,21,22] and three-dimensional (3D) traveling dust acoustic wave (DAW) with longitudinally oscillating dust particles charged and suspended in a low-pressure plasma [18]. For example, in the weakly disordered 3D DAW, RWEs tend to be preceded by the higher probability of surrounding lowamplitude holes (LAHs), coinciding with topological defect filaments [23,24], located at the troughs of modulation envelopes with null amplitudes and undefined phases. The crest surfaces surrounding LAHs are strongly distorted $[18,20,23,24]$. The additional transverse forces from distorted wave crests assist $3 \mathrm{D}$ particle focusing, which leads to RWEs generation [18].

With increasing driving, the single-scale weakly disordered wave can be changed to the wave turbulence state with more disordered extreme small- and large-amplitude events in multiscale waves, in which particles exhibit chaotic Lagrangian motion. Past studies mainly focused on the energy cascades and scaling of the continuous power spectra, multifractal and non-Gaussian dynamics, and wave mixing [1-3,5-7]. Certainly, it is intriguing to ask the following unexplored questions: Whether and how the above waveform focusing 
picture in the single-scale weakly disordered state can be extended to each mode in the wave turbulent state?

However, the lack of spectral gaps in the continuous power spectrum disables the spatiotemporal disentanglement of the wave turbulence into coupled multiscale modes using Fourier band-pass filtering. In the recent experimental study $[8,9]$, the multidimensional complementary ensemble empirical mode decomposition (MCEEMD), an adaptive decomposition method for analyzing nonstationary and nonlinear spatiotemporal data, was used to solve the above problem [25-27]. It demonstrated that each mode exhibits self-similar generic spatiotemporal dynamics [8,9], and the DAW turbulence can be viewed as a zoo of multiscale coherent excitations around LAHs [8].

Dust acoustic waves (DAWs), longitudinal waves composed of oscillating negatively charged micrometer-sized particles suspended in a low pressure plasma, are fundamental nonlinear acoustic-type density waves [28-30]. It can be self-excited through the interplay of dust inertia, screened Coulomb interactions, ion streaming, and background neutral gas collision. Governed by modulation-type nonlinear dynamical equations [28-31], it exhibits many ubiquitous nonlinear wave phenomenon, such as shocks [32], solitary waves [33], wave breaking [19], defects [20,23,24], rogue waves [18], wave turbulence [6-9], and percolation transition from ordered wave to wave turbulence [34]. Its proper spatiotemporal scales for large-area optical tracking of waveform evolution and individual particle motion at the discrete level, make it a good platform to construct spatiotemporal waveform dynamics in the $2+1 \mathrm{D}$ space-time space and a kinetic picture of wave-particle interaction for understanding waveform dynamics in nonlinear density wave systems.

In this work, using the DAW as a platform and MCEEMD, we further experimentally unravel the following unexplored issues for RWE generation in the acoustic-type wave turbulence: (a) for each mode, whether particle focusing by the distorted preceding waveform also leads to high-amplitude wave events in that mode, similar to that found in the previous RWE generation study in single-scale waves [18], (b) how particle focusing of various modes are correlated spatiotemporally, which leads to RWE generation, and (c) how multiscale LAHs are correlated with RWEs.

\section{EXPERIMENT}

As sketched in Fig. 1, the experiment is conducted in a cylindrical radio frequency dusty plasma system, the same as used in Ref. [8]. The weakly ionized glow discharge $\left(n_{e} \sim\right.$ $10^{9} \mathrm{~cm}^{-3}$ ) is generated in Ar gas at 220 mTorr (29.3 Pa) using a $14-\mathrm{MHz}$ RF power system with $2.3-\mathrm{W}$ RF power. A hollow square thin glass trap $25 \mathrm{~mm}$ in width and $25 \mathrm{~mm}$ in height is put on the center of the bottom electrode to confine the dusty plasma with MF (melamine-formaldehyde) particles $(5.3 \mu \mathrm{m}$ in diameter, $1.1 \times 10^{-10} \mathrm{~g}$ in mass, $1.5 \mathrm{~g} / \mathrm{cm}^{-3}$ in density), charged with about five thousands of electrons per particle. The estimated Debye length $\lambda_{D}$ is a few tens of micrometers. The downward propagating (along $-\mathbf{z}$ ) dust acoustic wave can be tuned from the self-excited plane wave state to the turbulent state by reducing background dissipation through lowering neutral gas pressure from 200 to 150 mTorr (26.6 to $20 \mathrm{~Pa}$ ).

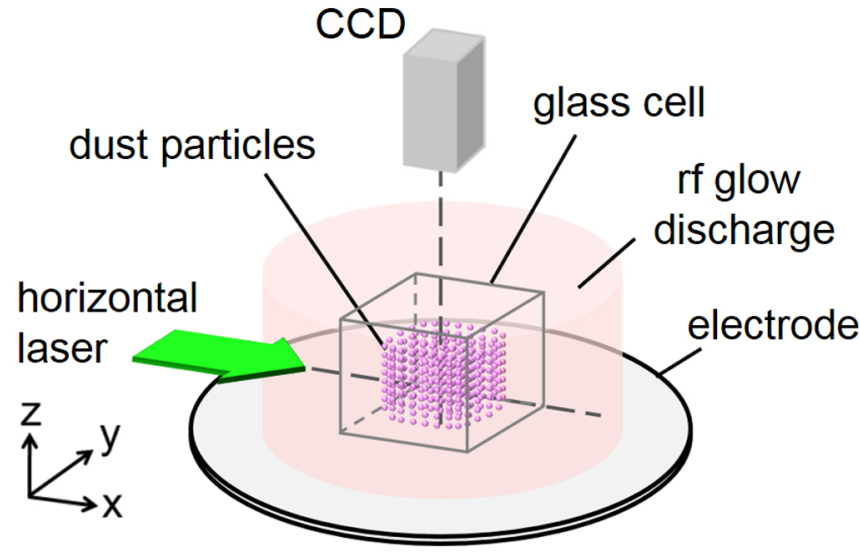

FIG. 1. Sketch of the experimental system, reproduced from Ref. [8].

The turbulent wave is evidenced by the irregular waveform with large-amplitude intermittent bursts and a continuous power-law decay spectrum [Figs. 2(a), 2(b), and 2(c)].

The dust image in the $x y$ plane illuminated by an expanded thin horizontal laser sheet is captured by a fast charge-coupled device (CCD) from the top. The normalized local dust density $n_{d}(x, y, t)$ can be obtained by measuring the coarse-grained image brightness $I_{d}$, normalized by its temporal average [8]. The RWE is identified as the wave height $H$ larger than $2 H_{S}$, where $H_{S}$ is the significant wave height, defined as the average of the top one third wave heights $[10,13]$, as shown in the histogram of the wave height in Fig. 2(c). The example in Fig. 2(d) showing the temporal evolution of $n_{d}-1$ illustrates how $H$ is defined. Here, 1 is the temporal average of $n_{d}$, and points $\mathrm{A}, \mathrm{C}, \mathrm{E}$, and $\mathrm{G}$ are the four sequential zero crossing points of $n_{d}-1$. Points $\mathrm{B}$ and $\mathrm{F}$ are the local minima for the region between $\mathrm{A}$ and $\mathrm{C}$ and the region between $\mathrm{E}$ and $\mathrm{G}$, respectively. Point $\mathrm{D}$ is the local maximum for the region between $\mathrm{C}$ and $\mathrm{E}$. The wave height $H$ for the region between $\mathrm{B}$ and $\mathrm{F}$ is defined as $\left[\left(n_{d D}-n_{d B}\right)+\right.$ $\left.\left(n_{d D}-n_{d F}\right)\right] / 2$. Namely, $H$ is defined as the average of the differences between the local maximum of $n_{d}$ to its two adjacent local minima of $n_{d}$. Here, the local maximum (minimum) is defined as the maximum (minimum) of the region of $n_{d}-1>0(<0)$, between two sequential zero crossing points of $n_{d}-1$.

Through the sifting process in MCEEMD based on EMD using Hilbert-Huang Transform [25] for a single time series, $n_{d}(t)$ from a local point in the $x y$ plane, is successively decomposed in terms of adaptively obtained, amplitude-frequency modulated oscillatory intrinsic mode function (IMF) with zero mean $n_{d j}(t)(j=1,2, \ldots, N)$, until reaching the residue $r(t)$ describing the mean trend [25-27]. Namely, $n_{d}(t)=$ $\sum_{j=1}^{N} n_{d j}(t)+r(t)$ and $n_{d j}=A_{j}(t) \cos \phi_{j}(t)$. From temporal variable $n_{d}(x, y, t)$ at all local points on the $x y$ plane, the single variable EMD is further expanded into the multidimensional EMD. The spatiotemporal evolution of the phase and the amplitude in different scales, i.e., $\phi_{j}(x, y, t)$ and $A_{j}(x, y, t)$ obtained from $n_{d j}(x, y, t)$, are used to characterize multiscale wave interaction and identify multiscale low-amplitude holes (LAHs). Here, the low-amplitude holes of decomposed mode 

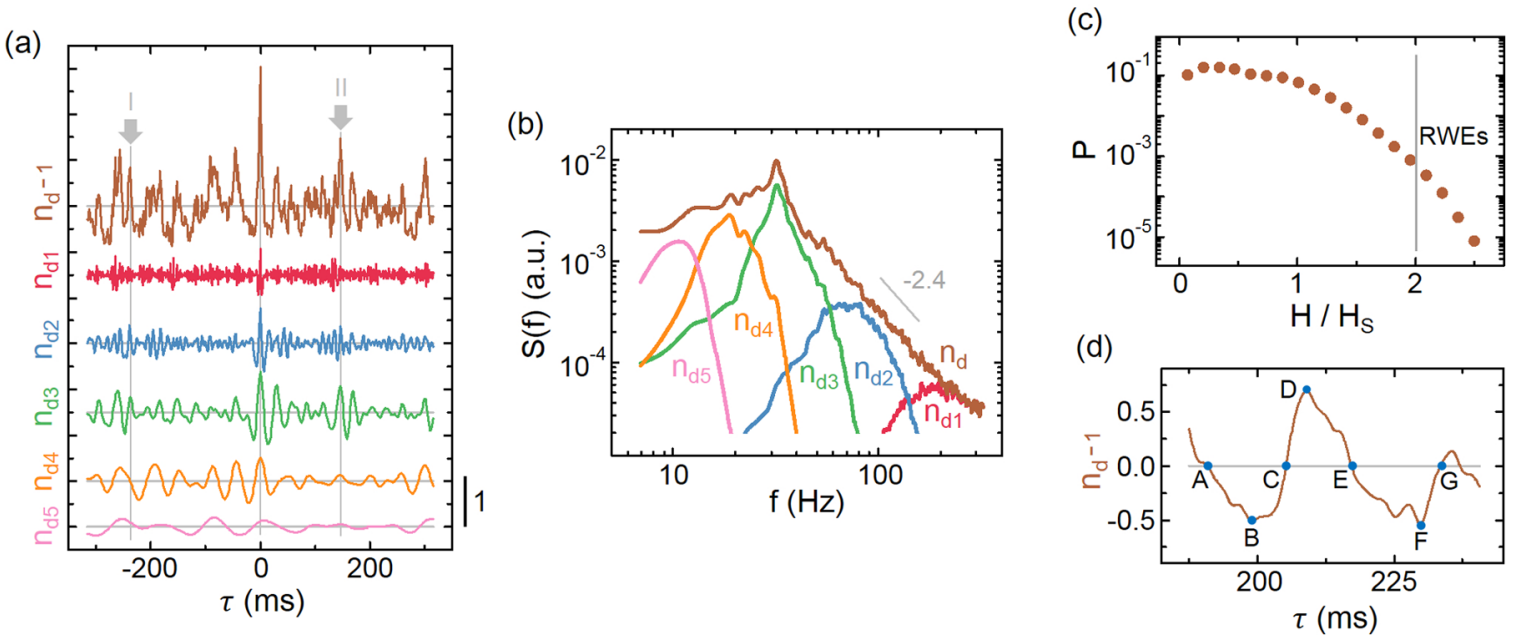

FIG. 2. (a) Temporal evolution of the typical localized dust density $n_{d}$ and its decomposed $n_{d j}$ around a RWE, in which the RWE peak times have been reset at $\tau=0$. The gray lines (in the middle of each oscillation curve) indicate the zero level. (b) Corresponding spatially averaged power spectra of (a) with scaling exponent -2.4 for the power-law decay. (c) Histogram of the ratio of wave height $H$ to significant wave height $H_{S}$, showing the existence of RWEs at $H \geqslant 2 H_{S}$. (d) Example of the temporal waveform of $n_{d}-1$, illustrating how $H$ is defined. Points $\mathrm{A}, \mathrm{C}, \mathrm{E}$, and $\mathrm{G}$ are the four sequential zero crossing points of $n_{d}-1$. Points $\mathrm{B}$ and $\mathrm{F}$ are the local minima for the region between $\mathrm{A}$ and $\mathrm{C}$ and the region between $\mathrm{E}$ and $\mathrm{G}$. Point $\mathrm{D}$ is the local maximum for the region between $\mathrm{C}$ and $\mathrm{E}$. Panels (a) and (b) are reproduced from Ref. [8].

$j$ are defined as the region with $A_{j}<1 \%$ of the spatiotemporally averaged $A_{j}$ [8]. More details of mode decomposition, especially through adding complementary noise sets for removing mode mixing, and the comparison of the results from several different methods based on empirical mode decomposition can be found in Refs. [26,27].

\section{RESULTS AND DISCUSSION}

Figure 2(a) shows the typical temporal evolution of $n_{d}$ and its decomposed IMFs after resetting the time for an RWE at $\tau=0$. The irregular amplitude and frequency modulations of all the IMFs cause the self-similar widely spread spectra of all IMFs in Fig. 2(b) and the large $n_{d}$ fluctuation in Fig. 2(a). Although the evolutions of amplitudes and phases of IMFs seem to be independent, there is a strong correlation between IMFs at the RWE. The emergence of the RWE is from the synchronization of the envelope peaks as well as the crests of all IMFs. The partial loss of the above synchronization leads to the reduction of wave height (e.g., indicated by arrows I and II respectively). Figure 3(a) shows the histograms of amplitude $A_{j}$ and phase $\phi_{j}$ of modes 1 to 5 , at the times for the large-amplitude events with $H>2 H_{S}$ (RWEs) and $=$ $1 H_{S}$. For all modes, the $A_{j}$ histograms shift rightward, and the $\phi_{j}$ histograms centered at $\phi_{j}=0$ become narrower, as $H$ increases. It leads to the increasing $\left\langle A_{j}\right\rangle$ (the ensemble average of $A_{j}$ ) and decreasing $\Delta \phi_{j}$ (the standard deviation of $\phi_{j}$ ), for all events with height $H$, with increasing $H$, respectively, shown in Fig. 3(b). The above findings statistically evidence that the increasing synchronization of the envelope peaks as well as the crests of IMFs is the key for the increasing $H$ and RWE generation.

For constructing a clear picture of the spatiotemporal waveforms leading to RWE formation, Fig. 4(a) shows the spatiotemporal waveform of $n_{d}(x, y, t)$ in the $x y t$ space.
Figure 4(b) shows the corresponding decomposed waveforms $n_{d j}(x, y, t)$ (top row) and the wave crest surfaces [isophase surfaces with $\phi_{j}(x, y, t)=0$ ] color coded by normalized wave amplitude $A_{j}(x, y, t) /\left\langle A_{j}(x, y, t)\right\rangle$ for major modes 2 to 4 , with main frequencies close to the center peak. The irregular spatiotemporal waveform modulations of those modes generate not only high-amplitude spots but also LAHs (filaments) coinciding with the topological defects [8]. They also lead to the turbulent waveform of $n_{d}(x, y, t)$ with intermittent bursts. As indicated by the red arrows (top) in Figs. 4(a) and 4(b), the RWE (the red disk region in which the wave height is higher than $2 H_{s}$ ) is localized in the $x y t$ space and coincides with the high $n_{d j}$ (brown) spots located in the wave crest surfaces for each mode in Fig. 4(b). Figure 4(c) more clearly illustrates that, by plotting together the top crest surfaces in the lower row of Fig. 4(b) colored coded by the mode number, the RWE occurs at the intersection of the three wave crests. Namely, the spatiotemporal phase synchronization of the large-amplitude spots of various modes leads to the emergence of the highly localized RWE in the $x y t$ space.

What is the correlation between multiscale LAHs and RWEs? Figures 5(a) and 5(b) show two typical examples of LAH filament distributions for the major modes 3 and 4 , color coded in green and brown [the lighter (darker) color represents the $+1(-1)$ topological charge], respectively, with RWEs located at the centers of the $x y t$ plots. Each RWE is surrounded by a core without LAHs, but the LAHs number is higher surrounding that core. To confirm that statistically, Fig. 6(a) further shows the normalized distribution functions $P_{j}(r, \tau)$ of finding LAHs of modes 1 to 5 with spatial and temporal separations $r$ and $\tau$, from crests with $H \geqslant 2,=1.5$ and $1 H_{S}$, where $\overline{\lambda_{j}}$ and $\overline{\tau_{j}}$ are the averaged wavelength and period of mode $j$, respectively. Note $\tau<(>) 0$ indicates that LAHs appear before (after) the large crest and $P_{j}(r, \tau)=1$ is the averaged LAH density. 

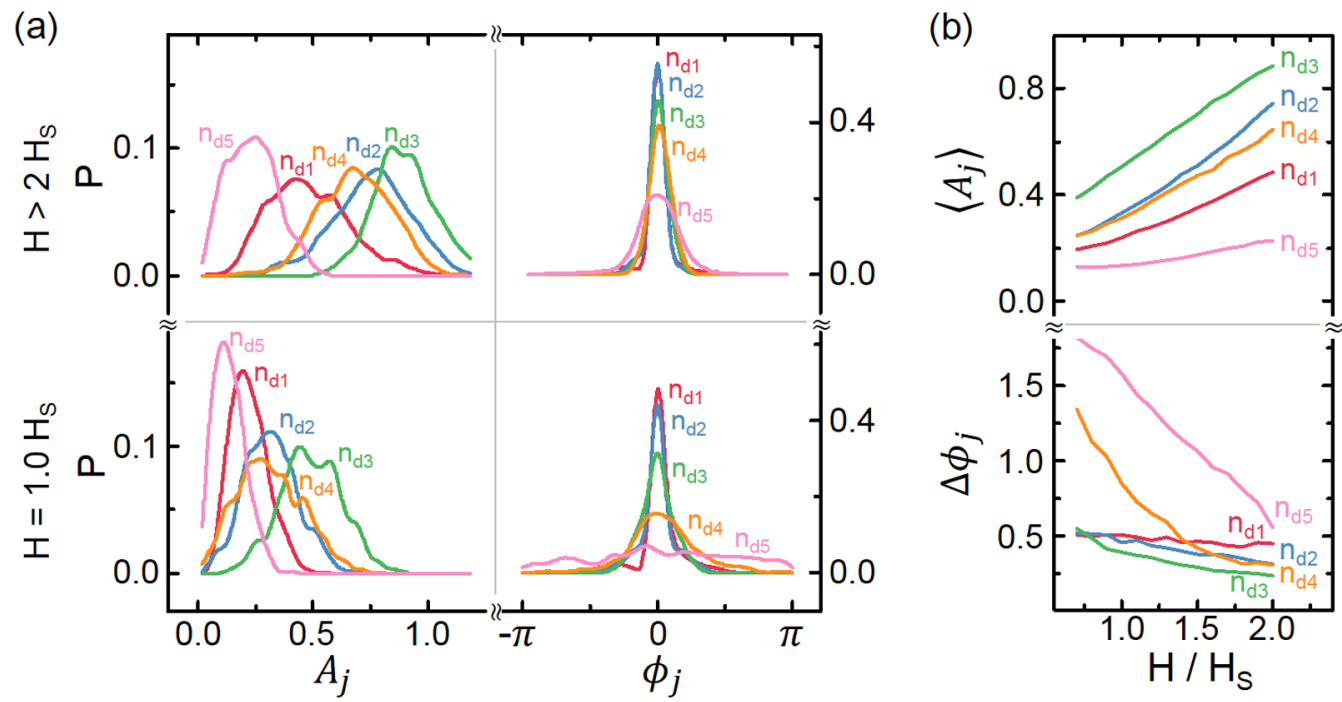

FIG. 3. (a) Histograms of amplitude $A_{j}$ and phase $\phi_{j}$ of modes 1 to 5 at times for $H=2 H_{S}$ and $1 H_{S}$ events. (b) The ensemble average of $A_{j}\left(\left\langle A_{j}\right\rangle\right)$ and the standard deviation of $\phi_{j}\left(\Delta \phi_{j}\right)$ vs the ratio of wave height $H$ to the significant wave height $H_{S}$.

The dark blue semisphere regions with $\left|\tau / \overline{\tau_{j}}\right|$ and $r / \overline{\lambda_{j}}<$ 1 for $H / H_{s}>2$, indicate that $P_{j}$ for all modes are very small. These regions coincide with the regions of high $A_{j}$ (wave amplitude) regions in Fig. 6(b). It means that the spatiotemporal synchronization of localized highamplitude regions of all major modes for RWE generation tend to exclude LAHs to the orange regions surrounding the dark blue semisphere regions with $P_{j}>1$. Those nonuniform distributions of $P_{j}$ are gradually smoothed out with decreasing $H$.
What are the physical origins for the above observations? The previous RWE study for the single-scale DAW also indicates the similar behaviors of $P_{j}$ distribution in the $\tau / \overline{\tau_{j}}, r / \overline{\lambda_{j}}$ space [18]. The following picture of particle compression and focusing by the preceding surrounding waveform was used to explain the RWE generation. Particles are negatively charged. In the moving frame of a traveling DAW, particles can be decelerated and accelerated by the electric field normal to the contour of the spatial dust density distribution, while entering and leaving the crest front and crest rear respectively. The

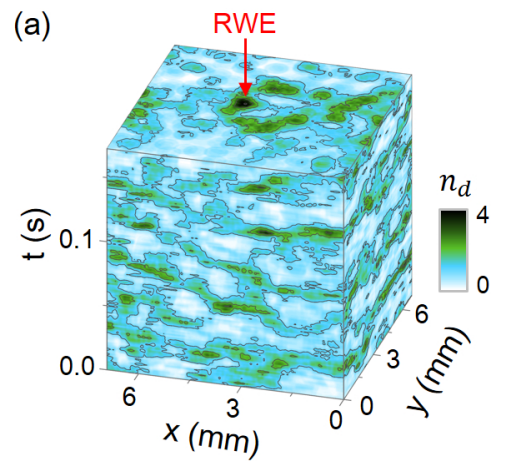

(b)
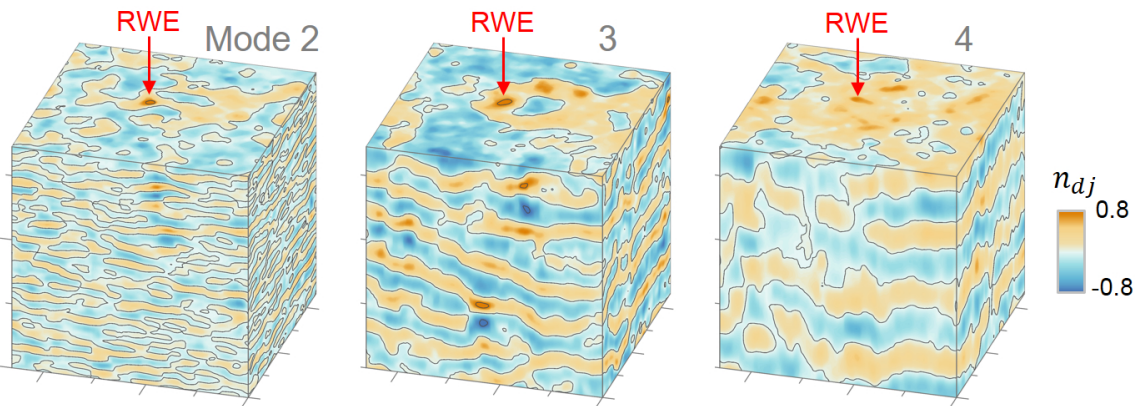

(c)
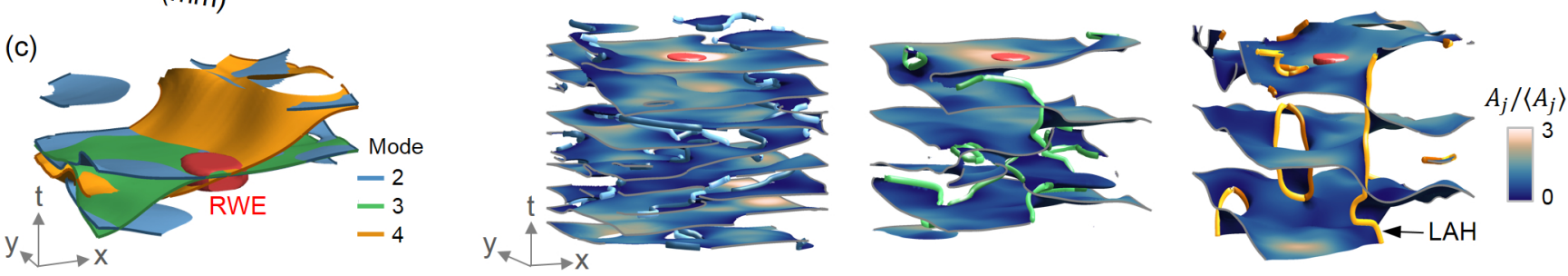

FIG. 4. (a) Turbulent waveforms color coded by $n_{d}$ on the $x y, x t$, and $y t$ planes. The red arrow (top) labels the RWE (the dark region), localized on the top $x y$ plane. (b) Top row: the corresponding decomposed waveforms $n_{d j}$ of the three major modes 2 to 4 . The plots share the same scales as those in panel (a). Bottom row: stereo wave crest surfaces [isophase surfaces with $\phi_{j}(x, y, t)=0$ ] surrounding the RWE (red disk region) color coded by normalized wave amplitude $A_{j}(x, y, t) /\left\langle A_{j}(x, y, t)\right\rangle$. The lengths of gray arrows (bottom left) indicating $x$ and $y$ axes correspond to $0.5 \mathrm{~mm}$. The length of the gray arrow indicating the $t$ axis corresponds to $13 \mathrm{~ms}$. The LAH filaments with lighter and darker colors in each panel coincide with the defect filaments with +1 and -1 topological charges, respectively. (c) Coplot of the wave crest surfaces for modes 2 to 4 . Phase synchronization of modes 2 to 4 occurring at the intersection of the three wave crests leads to RWE generation. 

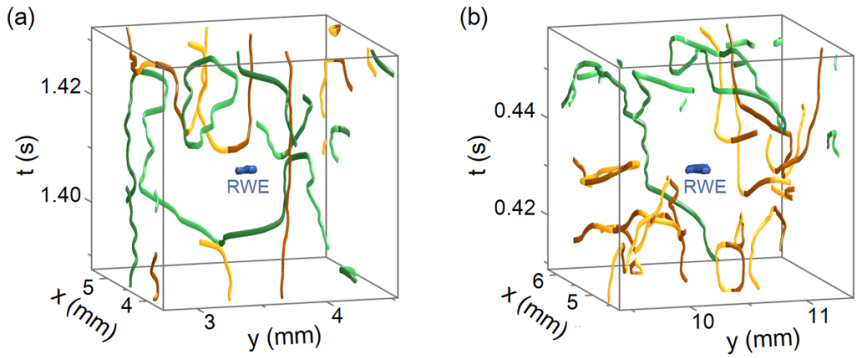

FIG. 5. [(a), (b)] Two typical examples of LAH filament distributions for the major modes 3 and 4, color coded in green and brown respectively, with RWEs located at the centers of the $x y t$ plots. The lighter and darker colors represent the +1 and -1 topological charges, respectively.

distorted preceding crest rear near LAHs can cause particle focusing of the leaving particles and the subsequent strong compression and accumulation when those particles enter the trailing wave crest. It is the key for RWE generation in the single-scale DAW and explains why the RWE tends to be led by the higher probability of LAHs.

In order to test whether this picture can be further extended to the multiscale wave turbulence, Fig. 7(a) gives an example showing the distorted waveforms of the major modes 3 and 4 preceding an RWE in the $x t$ plane. Crosses indicate defect locations and vectors show the forces (along the dust density gradient direction) exerted on particles from the wave field. We further measure the focusing parameter $F_{j}$ which characterize the degree of particle focusing by the preceding surrounding waveform of each mode for the highamplitude events. The focusing parameter is calculated as $F_{j}=\iint_{S}-\nabla n_{d j} \cdot \hat{n} d S$, where $S$ is the preceding crest surface within one averaged wavelength and one averaged period of mode $j$ and $\hat{n}$ is the unit vector pointing from the position of the preceding crests to the position of target crest event. Figure 7(b) shows that for each mode, $F_{j}$ for RWEs $(H>$ 2) are greater than that for the events with $H=1.0 H_{S}$.

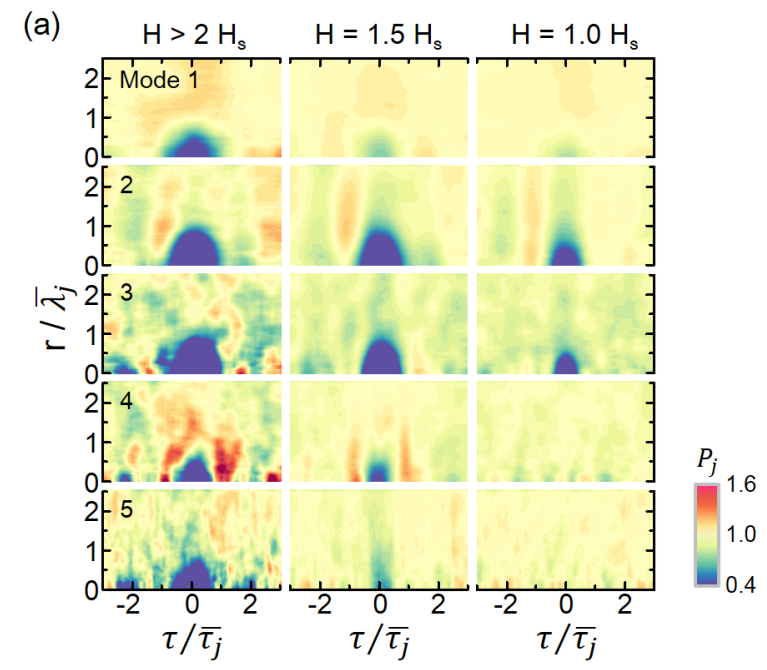

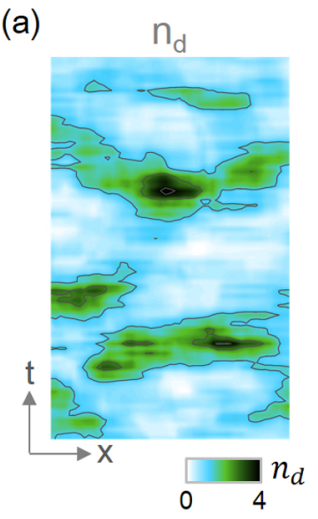
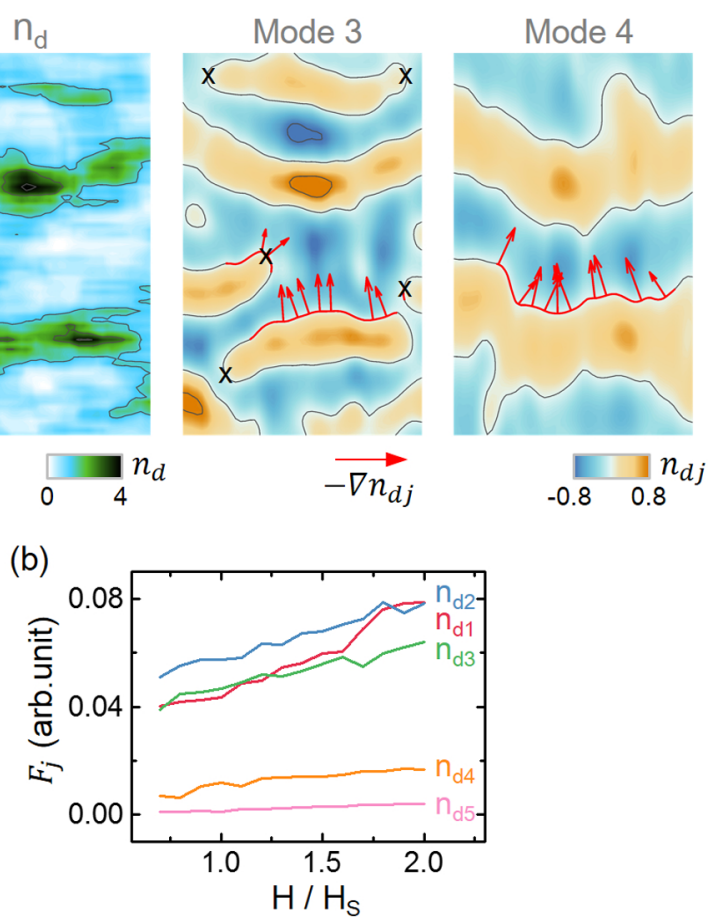

FIG. 7. (a) Contour plot of $n_{d}$ and the corresponding decomposed $n_{d j}$ around a RWE in the $x t$ plane. The red arrows (in contour plots) indicate the magnitude and direction of $-\nabla n_{d j}$ which is proportional to the forces exerted on particles. The lengths of gray arrows (bottom left) indicating $x$ and $t$ axes correspond to $1 \mathrm{~mm}$ and $25 \mathrm{~ms}$, respectively. The black crosses indicate the locations of defects (LAHs). (b) The focusing parameter $F_{j}$ of each mode $j$ versus $H / H_{s}$.

The above results suggest that, down to each mode, particle focusing by the distorted preceding waveforms for RWE generation still plays an important role for the multiscale wave turbulence. Note that $F_{4}$ and $F_{5}$ are much smaller than other $F_{j}$. It implies that RWE generation is mainly contributed by

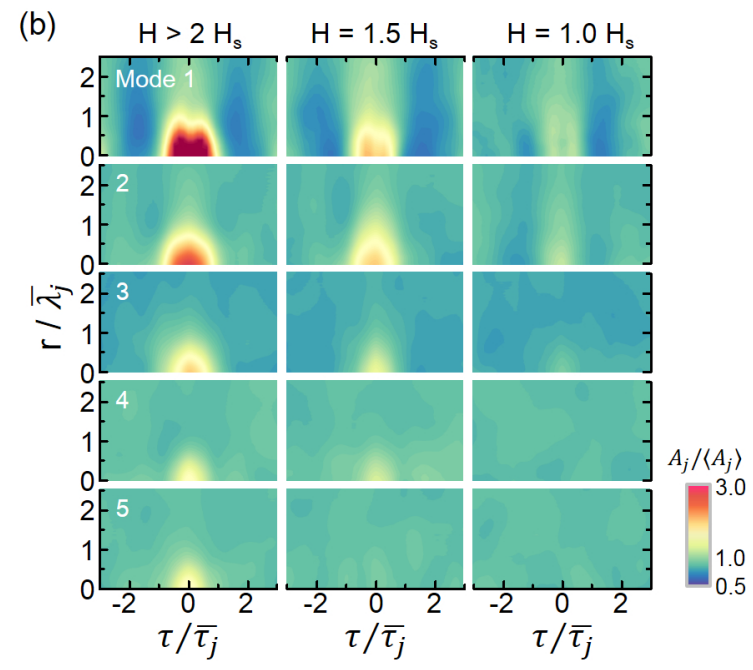

FIG. 6. [(a), (b) Contour plots of the normalized distribution function $P_{j}(r, \tau)$ of finding LAHs of mode $j$ and normalized amplitude distribution $A_{j}(x, y, t) /\left\langle A_{j}(x, y, t)\right\rangle$, with spatial and temporal separations $r$ and $\tau$, from crests with $H \geqslant 2,=1.5$ and $1 H_{S}$, where $\overline{\lambda_{j}}$ and $\overline{\tau_{j}}$ are the averaged wavelength and period of mode $j$, respectively. 
the focusing of short-wavelength (small- $j$ ) modes, since the local electric forces which increases with density gradients are small for the long wavelength modes 4 and 5 .

Note that, for the RWE generation mechanism, unlike the formation of envelope soliton through modulational instability in 1D nonlinear waves, curved focusing in the water surface wave and DAW have been demonstrated as the mechanism for the higher dimensional nonlinear waves [14,18,21,22]. However, those higher dimensional studies were limited in single scaled weakly disordered waves. Our study demonstrates that this focusing idea can be further extended to the multiscale wave turbulence of the nonlinear wave, once focusing from various modes are synchronized. Our analytical methods such as decomposing wave turbulence through MCEEDM for the spatiotemporal behaviors of different scale modes, and using the focusing parameter to identify the degree of wave front focusing of different scale modes, could also be applied in other wave turbulence studies.

\section{CONCLUSION}

In conclusion, through MCEEMD, we have investigated the rogue wave generation in dust acoustic wave turbulence with multiscale wave excitations and constructed a picture of RWE generation through synchronization of particle focusing by the distorted waveform at different scales. At the RWE, the mean amplitudes and phase distributions of multiscale modes are larger and narrower around zero-deg phases, respectively, than those at other normal wave height events. In the $2+1 \mathrm{D}$ spatiotemporal space, RWEs are located at the intersection of distorted multiscale crest surfaces with large amplitudes and proceeded by the higher probability of finding low-amplitude holes in each mode. It indicates that RWEs are associated with the spatiotemporal phase synchronization of large-amplitude crests of various modes. The 3D particle focusing by the synchronized multiscale distorted crests nearby multiscale low-amplitude holes is the key for RWE generation in 3D multiscale DAW turbulence.

Our findings and the analytical methods of the synchronization of multiscale waveform focusing shed light on the extreme event generation in the mechanical wave turbulence of various nonlinear media. The study paves a new way for other future studies in different nonlinear wave systems.

\section{ACKNOWLEDGMENT}

This work is supported by the Ministry of Science and Technology, Taiwan, under Contract No. MOST-105-2112M-008-005-MY3.
[1] E. Falcon, C. Laroche, and S. Fauve, Phys. Rev. Lett. 98, 094503 (2007).

[2] H. Punzmann, M. G. Shats, and H. Xia, Phys. Rev. Lett. 103, 064502 (2009).

[3] S. Coulibaly, M. Taki, A. Bendahmane, G. Millot, B. Kibler, and M. G. Clerc, Phys. Rev. X 9, 011054 (2019).

[4] A. S. Mikhailov and K. Showalter, Phys. Rep. 425, 79 (2006).

[5] A. N. Ganshin, V. B. Efimov, G. V. Kolmakov, L. P. MezhovDeglin, and P. V. E. McClintock, Phys. Rev. Lett. 101, 065303 (2008).

[6] J. Pramanik, B. Veeresha, G. Prasad, A. Sen, and P. Kaw, Phys. Lett. A 312, 84 (2003).

[7] Y.-Y. Tsai, M.-C. Chang, and L. I, Phys. Rev. E 86, 045402(R) (2012).

[8] P.-C. Lin and L. I, Phys. Rev. Lett. 120, 135004 (2018).

[9] H.-W. Hu, W. Wang, and L. I, Phys. Rev. Lett 123, 065002 (2019).

[10] C. Kharif, E. Pelinovsky, and A. Slunyaev, Rogue Waves in the Ocean (Springer, Berlin, 2009).

[11] M. Onorato, A. R. Osborne, M. Serio, and L. Cavaleri, Phys. Fluids 17, 078101 (2005).

[12] M. Shats, H. Punzmann, and H. Xia, Phys. Rev. Lett. 104, 104503 (2010).

[13] A. Chabchoub, N. P. Hoffmann, and N. Akhmediev, Phys. Rev. Lett. 106, 204502 (2011).

[14] H.-Y. Chen, C.-Y. Liu, and L. I, Phys. Rev. Fluids 3, 064401 (2018).

[15] D. R. Solli, C. Ropers, P. Koonath, and B. Jalali, Nature (London) 450, 1054 (2007).

[16] C. J. Gibson, A. M. Yao, and G.-L. Oppo, Phys. Rev. Lett. 116, 043903 (2016).
[17] H. Bailung, S. K. Sharma, and Y. Nakamura, Phys. Rev. Lett. 107, 255005 (2011).

[18] Y.-Y. Tsai, J.-Y. Tsai, and L. I, Nat. Phys. 12, 573 (2016).

[19] L.-W. Teng, M.-C. Chang, Y.-P. Tseng, and L. I, Phys. Rev. Lett. 103, 245005 (2009).

[20] J.-Y. Tsai, Y.-Y. Tsai, and L. I, Phys. Plasmas 22, 013701 (2015).

[21] C. Brandini and S. T. Grilli, in Proceedings of the 4th International Symposium on Ocean Wave Measurement and Analysis, edited by B. L. Edge and J. M. Hemsley (American Society of Civil Engineers, 2001), pp. 1102-1111.

[22] C. Fochesato, S. Grilli, and F. Dias, Wave Motion 44, 395 (2007).

[23] M.-C. Chang, Y.-Y. Tsai, and L. I, Phys. Plasmas 20, 083703 (2013).

[24] Y.-Y. Tsai and L. I, Phys. Rev. E 90, 013106 (2014).

[25] N. E. Huang, Z. Shen, S. R. Long, M. C. Wu, H. H. Shih, Q. Zheng, N.-C. Yen, C. C. Tung, and H. H. Liu, Proc. R. Soc. London, Ser. A 454, 903 (1998).

[26] Z. Wu and N. E. Huang, Adv. Adapt. Data. Anal. 01, 1 (2009).

[27] J.-R. Yeh, J.-S. Shieh, and N. E. Huang, Adv. Adapt. Data. Anal. 02, 135 (2010).

[28] N. N. Rao, P. K. Shukla, and M. Y. Yu, Planet. Space Sci. 38, 543 (1990).

[29] P. Kaw and R. Singh, Phys. Rev. Lett. 79, 423 (1997).

[30] P. K. Shukla and B. Eliasson, Rev. Mod. Phys. 81, 25 (2009).

[31] W. M. Moslem, R. Sabry, S. K. El-Labany, and P. K. Shukla, Phys. Rev. E 84, 066402 (2011).

[32] J. Heinrich, S.-H. Kim, and R. L. Merlino, Phys. Rev. Lett. 103, 115002 (2009).

[33] P. Bandyopadhyay, G. Prasad, A. Sen, and P. K. Kaw, Phys. Rev. Lett. 101, 065006 (2008).

[34] P. C. Lin and L. I, Phys. Plasmas 27, 010703 (2020). 